\title{
La flora leñosa establecida luego de las quemas en el valle de Chanchamayo - Selva central del Perú
}

\author{
The woody flora established after burning in the Chanchamayo Valley \\ - Rainforest of central Peru
}

Esaú Echía ${ }^{1, *}$, Carlos Reynel ${ }^{1}$ y María Manta ${ }^{2}$

Recibido: 07 mayo 2018 | Aceptado: 12 junio 2019 | Publicado en línea: 30 junio 2019 Citación: Echía, E; Reynel, C; Manta, M. 2019. La flora leñosa establecida luego de las quemas en el valle de Chanchamayo - Selva central del Perú. Revista Forestal del Perú 34(1): 83-101. DOI: http:// dx.doi.org/10.21704/rfp.v34i1.1287

\begin{abstract}
Resumen
En las últimas décadas los incendios forestales producidos en la Selva central de Perú se han incrementado, causando efectos en la vegetación. A través del tiempo estás áreas afectadas por el fuego se han ido regenerando. Sin embargo, informaciones de la riqueza y composición florística son escasas en esta región. En el presente estudio evaluamos la flora leñosa en áreas afectadas por el fuego en tres edades aproximadas de bosque (5, 10 y 25 años). Instalamos 9 transectos de $2 \times 500 \mathrm{~m}$ ( $0.1 \mathrm{ha})$; donde registramos todos los individuos con DAP (medido a $1.3 \mathrm{~m}$ del suelo) $\geq 2,5 \mathrm{~cm}$. Los resultados muestran que los bosques de 5, 15 y 25 años presentan a las siguientes familias más abundantes: Asteraceae y Euphorbiaceae; Fabaceae y Asteraceae; y Malpighiaceae y Piperaceae respectivamente; las especies más abundantes fueron: Vernonanthura patens (Kunth) H.Rob, Acalypha sp., y Celtis pubescens Spreng.; Machaerium inundatum (Mart. ex Benth.) Ducke, Dendrophorbium sp. y Trema micrantha (L.) Blume; y Heteropterys laurifolia (L.) A. Juss., Piper aduncum L. y Allophylus floribundus (Poepp.) Radlk. respectivamente. Asimismo, los resultados muestran que los bosques de 5, 15 y 25 años poseen 36, 95 y 220 individuos; 6, 12 y 36 especies; 6, 11 y 34 géneros; 4, 10 y 23 familias.
\end{abstract}

Palabras clave: incendios forestales, riqueza, composición, bosque secundario, transectos

\footnotetext{
${ }^{1}$ Herbario Forestal MOL, Facultad de Ciencias Forestales, Universidad Nacional Agraria La Molina (UNALM), Av. La Molina s/n, La Molina, Lima, Perú.

${ }^{2}$ Departamento Académico de Manejo Forestal, Facultad de Ciencias Forestales, Universidad Nacional Agraria La Molina (UNALM), Av. La Molina s/n, La Molina, Lima, Perú.

* Autor de Correspondencia: esaua.er@gmail.com
} 
La flora leñosa establecida luego de las quemas en el valle de

\begin{abstract}
On the last decades, forest fires in the rainforest of Central Peru have been increasing, causing effects on the forest's vegetation. The affected areas have gone regenerating. However, information about richness and floristic composition are scarce in this region. In the current study we assessed the woody flora in forests of three ages (5, 10 and 25 years) affected by forest fires and installed 9 transects $(2 \times 500 \mathrm{~m})$; individuals with a DBH (measured at $1.3 \mathrm{~m}$ from the ground) $\geq 2.5 \mathrm{~cm}$ were measured. In the forests of 5, 10 and 25 years it was found the following: the more abundant families were Asteraceae and Euphorbiaceae; Fabaceae and Asteraceae; and Malpighiaceae and Piperaceae respectively; and more abundant species were Vernonanthura patens (Kunth) H.Rob, Acalypha sp., y Celtis pubescens Spreng.; Machaerium inundatum (Mart. ex Benth.) Ducke, Dendrophorbium sp. y Trema micrantha (L.) Blume; y Heteropterys laurifolia (L.) A. Juss., Piper aduncum L. y Allophylus floribundus (Poepp.) Radlk. respectively. Likewise, it was found 36, 95 and 220 individuals on average; 6, 12 and 36 species; 6, 11 and 34 genera; 4, 10 and 23 families for the forest of 5, 15 and 25 years, respectively.
\end{abstract}

Wey words: forest fires, richness, composition, secondary forest, transects

\section{Introducción}

La protección de los bosques naturales se ha convertido en una urgente prioridad no sólo por la diversidad biológica sino debido a los servicios ecosistémicos que brinda al hombre, tales como la fijación de $\mathrm{CO}_{2}$, la regulación de la temperatura, pero sobre todo por su papel en el ciclo hidrológico y así en la disponibilidad de agua dulce para el hombre y ecosistemas (Manta 2007). La destrucción de los bosques tropicales ha recibido atención mundial debido a que estos ecosistemas tienen un papel único en términos ecológicos y, sobretodo, la incesante amenaza a su existencia, con efectos directos sobre las emisiones netas de carbono debidas a la deforestación y degradación; asimismo, las tasas de deforestación están lejos de ser uniformes en todo el mundo y dependen de los diferentes análisis y fuentes de datos usados para su cálculo (Armenteras y Rodríguez 2014).

FAO (2011) estima una deforestación neta a nivel mundial de $0.20 \%$ en la década que va de 1990 a 2000, $0.12 \%$ entre el 2000 y el 2005 y $0.14 \%$ entre el 2005 y el 2010 , con una pérdida neta de 5.2 millones de hectáreas en la década del 2000 al 2010 (Armenteras y Rodríguez 2014).

Los incendios forestales propician cambios globales que afectan a la biósfera, y se les considera la tercera causa en la pérdida y degradación de grandes extensiones de terrenos forestales; y son una fuente significativa de las emisiones de gases que provocan el calentamiento global (Martínez et al. 2014). Los incendios forestales que ocurren en el Perú son escasamente estudiados y son políticamente aceptados como quemas agropecuarias, ya que se otorga la propiedad del suelo forestal a quien cultiva los suelos forestales y de protección con productos alimenticios para el hombre o el ganado (Manta y León 2004). Las quemas agropecuarias o incendios forestales son explícitamente originadas por el hombre y obedecen a diferentes motivaciones. Sin embargo, el fuego se escapa del terreno agropecuario y se propaga sin control y se extiendan sobre las tierras forestales cubiertas de bosques. Por ejemplo, en selva central de Perú (Satipo) se incendiaron 50,000 ha de bosques (Manta 2005).

En años recientes, el estudio de la diversidad biológica - biodiversidad, la complejidad del componente viviente, de la flora y la fauna se ha consolidado como una de las líneas más importantes y de mayor valor aplicado entre los estudios ecológicos de las zonas de bosque tropical (Reynel y Antón 2004). Sin embargo, estudios realizados sobre la regeneración en bosques tropicales después de quemas aún es escasa, siendo los estudios más próximos los realizados y relacionados por Cáceres (2005) 
y Quintero (2017). La falta de información de especies hace importante realizar evaluaciones para tener conocimiento y generar posibles métodos, técnicas y/o desarrollo de planes de rehabilitación de zonas devastadas por los incendios.

En ese sentido, el objetivo del presente estudio fue determinar la riqueza y composición florística de bosques de 5, 10 y 25 años, regenerados después de quemas en el valle de Chanchamayo, departamento de Junín (Perú).

\section{Materiales y Métodos}

\section{Localización}

La zona de estudio se localizó en el Fundo "La Génova" perteneciente al IRD selva de la Universidad Nacional Agraria La Molina (UNALM), en la Selva Central del Perú, departamento de Junín entre los distritos de San Ramón y La Merced, al lado opuesto del río Chanchamayo y ubicado aproximadamente entre las coordenadas UTM 8771,500-
$8774,500 \mathrm{~N}$ y $459,500-463,500 \mathrm{~W}$ Zona $18 \mathrm{~S}$ (Llave 2008) (Figura 1).

Para localizar los transectos de estudio se hizo un recorrido general por el Instituto Regional de Desarrollo (IRD) - UNALM en el Fundo "La Génova", con el apoyo del Técnico Forestal Alejandro Camarena Segura, trabajador conocedor del área del fundo y que ha permanecido en la zona desde hace más de 30 años, se logró identificar bosques que habían sufrido de quemas recurrentes y que ahora se encuentran en regeneración y sucesión. En total se precisaron tres edades de bosque: 5,10 y 25 años de regeneración y sucesión desde la última quema (Figura 2).

\section{Metodología}

Se emplearon Transectos Gentry, que consisten en líneas de $2 \mathrm{~m}$ de ancho por $500 \mathrm{~m}$ de largo, con una superficie de 0,1 ha, donde se registraron y colectaron todas las plantas mayores a $2,5 \mathrm{~cm}$ de DAP (Gentry 1982). Se establecieron en total tres transectos por cada

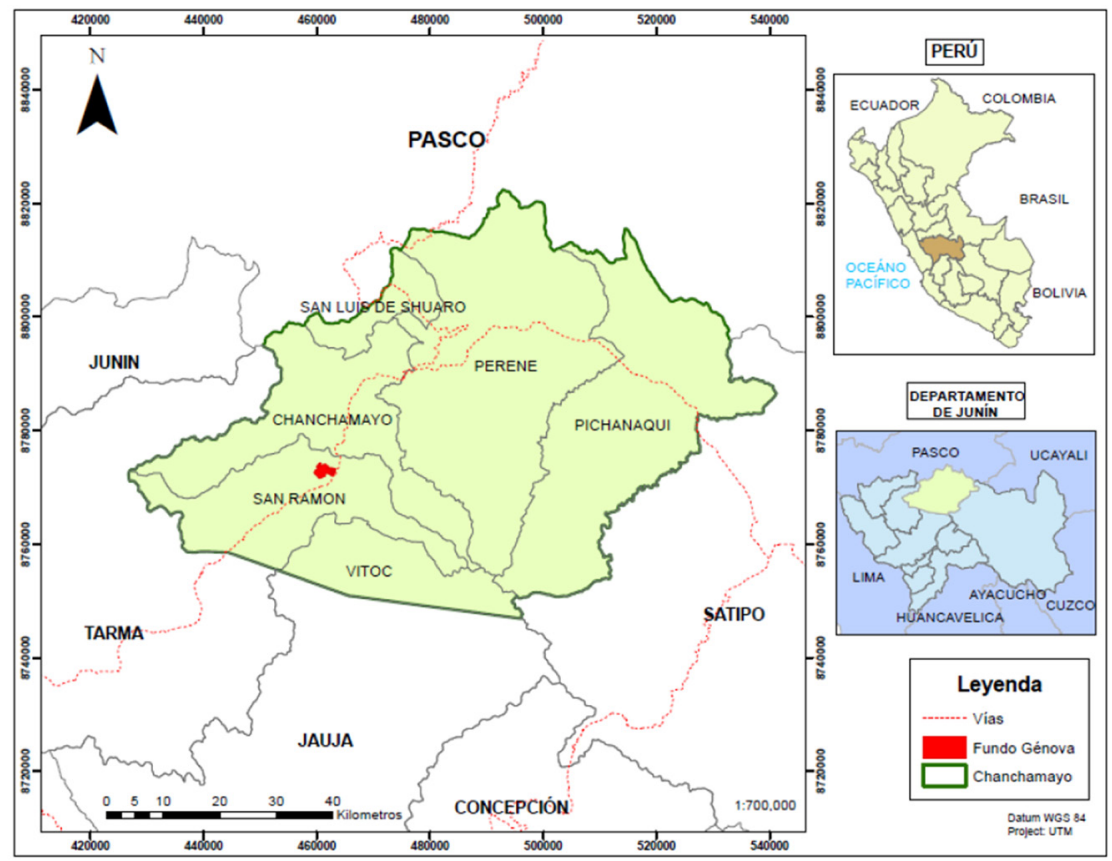

Figura 1. Ubicación de la zona de estudio. 
La flora leñosa establecida luego de las quemas en el valle de

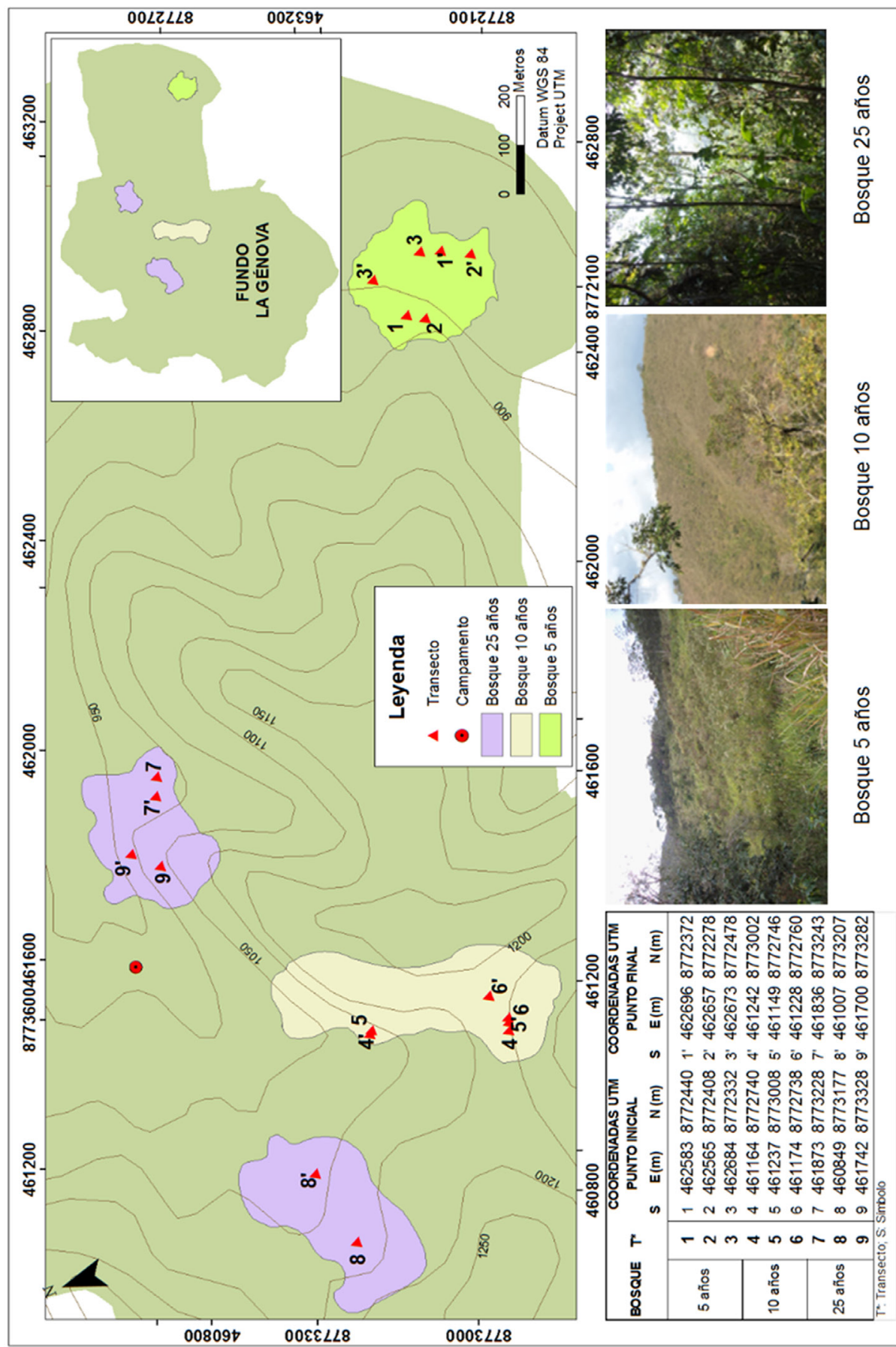

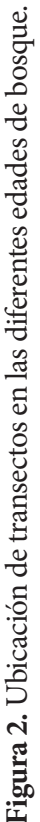


edad de bosque (Figura 2). Previamente fue elaborado un mapa de las áreas de bosques secundarios de diferentes edades que luego fue cuadriculado y se eligieron al azar cuadriculas sobre las cuales se ubicó el inicio de cada transecto.

La evaluación de la vegetación fue realizada en el periodo 2012-2013. Los puntos iniciales y finales de cada transecto en las diferentes edades de bosque fueron georreferenciados (Figura 2). Una vez georreferenciado el primer punto del transecto se empezó a abrir trocha de una distancia de $50 \mathrm{~m}$ para el primer sub-transecto (de $2 \mathrm{~m}$ de ancho por $50 \mathrm{~m}$ de largo), y así sucesivamente hasta completar los $500 \mathrm{~m}$ (se tuvo consideración que los transectos fueron establecidos dentro del mismo tipo de bosque). Se evaluó el diámetro y la altura de la vegetación existente a lo largo de todo el transecto $(2 \times 500 \mathrm{~m})$. Las mediciones del diámetro se realizaron a la altura del pecho (DAP) incluyendo a todos los individuos con un diámetro $\geq$ $2.5 \mathrm{~cm}$ o una longitud de circunferencia $\geq 7.8$ $\mathrm{cm}$ haciendo uso de una cinta métrica. Las colecciones botánicas y secado de las muestras fueron desarrollados de acuerdo al proceso de herborización descrito por Bridson y Forman (1998).

La identificación de las especies fue realizada en el Herbario Forestal MOL (Facultad de Ciencias Forestales - UNALM). Se emplearon las colecciones de referencia existentes en el Herbario y se consultó bibliografía especializada incluyendo Macbride (1936-1971), Meneses (1989), Spichiger et al. (1990), De La torre (2002), Pino (2002), Cáceres (2004), Silva (2005), Reynel (2007) y Reynel (2016) y sus correspondientes claves de identificación. El sistema de clasificación utilizado fue el de APG IV (2016).

\section{Resultados}

\section{Riqueza y Composición Florística}

En los tres bosques evaluados $(5,10$ y 25 años) se registraron en total 82 especies, 71 géneros y 35 familias. En las áreas de bosque de 5 años se registró 13 especies, 13 géneros y
7 familias. En las áreas de bosque de 10 años se registró 21 especies, 20 géneros y 14 familias. Y en las áreas de bosque de 25 años se registró 70 especies, 62 géneros y 34 familias (Cuadro 1).

Las familias con más géneros fueron Astereaceae (10), Rubiaceae (06); Moraceae (05), y Urticaceae (04); los géneros con mayor cantidad de especies fueron Piper (04), Ficus (03), Machaerium (02), Terminalia (02), Ocotea (02), Eugenia (02) y Solanum (02). Aquellas familias y géneros que aparecieron en las tres edades de bosque fueron Euphorbiaceae - Acalypha; Asteraceae - Vernonanthura; Fabaceae Machaerium; Urticaceae - Cecropia y Piperacae - Piper.

\section{Abundancia}

En las áreas de bosque secundario originalmente sometidas a quema, las familias más abundantes para los bosques de 5, 10 y 25 años fueron Asteraceae (65) y Euphorbiaceae (09); Fabaceae (103) y Asteraceae (50); Malpighiaceae (75) y Piperaceae (65) respectivamente. En cuanto a los géneros más abundantes fueron Vernonanthura y Acalypha; Machaerium y Dendrophorbium; Piper y Heteropterys respectivamente, mientras que las especies más abundantes fueron Vernonanthura patens (Kunth) H. Rob. y Acalypha sp.; Machaerium inundatum (Mart. ex Benth.) Ducke y Dendrophorbium sp.; Heteropterys laurifolia (L.) A. Juss. y Piper aduncum L. En los Anexos 1 al 4 se detalla la abundancia hallada para las diferentes edades de bosque.

Fueron registrados en total 108 individuos para la edad de 5 años, 285 individuos para la edad de 10 años y 659 individuos para la edad de 25 años.

\section{Estructura}

En la distribución diamétrica, para el bosque de 5 años, cerca del $97 \%$ de los individuos presentan un diámetro entre 2.5 y $5 \mathrm{~cm}$, siendo Erythrina ulei Harms $(10.2 \mathrm{~cm})$ la especie de mayor diámetro. Para el bosque de 10 años, el $96.84 \%$ de los individuos se encuentran entre 2.5 y $7.5 \mathrm{~cm}$ de diámetro, siendo Cecropia polystachya Trécul $(17.1 \mathrm{~cm})$ la especie de 


\begin{tabular}{|c|c|c|c|c|c|c|c|c|c|}
\hline Bosque & \multicolumn{3}{|c|}{5 años } & \multicolumn{3}{|c|}{10 años } & \multicolumn{3}{|c|}{25 años } \\
\hline Riqueza & 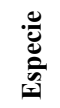 & تٍِّّ & 丞 & 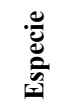 & نัّ & : & 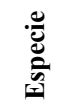 & & \\
\hline Transecto i & 9 & 9 & 5 & 13 & 12 & 10 & 33 & 32 & 25 \\
\hline Transecto ii & 4 & 4 & 3 & 11 & 11 & 9 & 40 & 36 & 23 \\
\hline Transecto iii & 5 & 5 & 4 & 11 & 11 & 10 & 36 & 34 & 20 \\
\hline Total absoluto & 13 & 13 & 7 & 21 & 20 & 14 & 70 & 62 & 34 \\
\hline Desviación estándar & 2.65 & 6 & 1.00 & 1.15 & 11 & 0.58 & 3.51 & 34 & 2.52 \\
\hline Promedios & 6 & 2.65 & 4 & 12 & 0.58 & 10 & 36 & 2.00 & 23 \\
\hline
\end{tabular}

Cuadro 1. Riqueza en diferentes edades de bosque (5, 10 y 25 años).

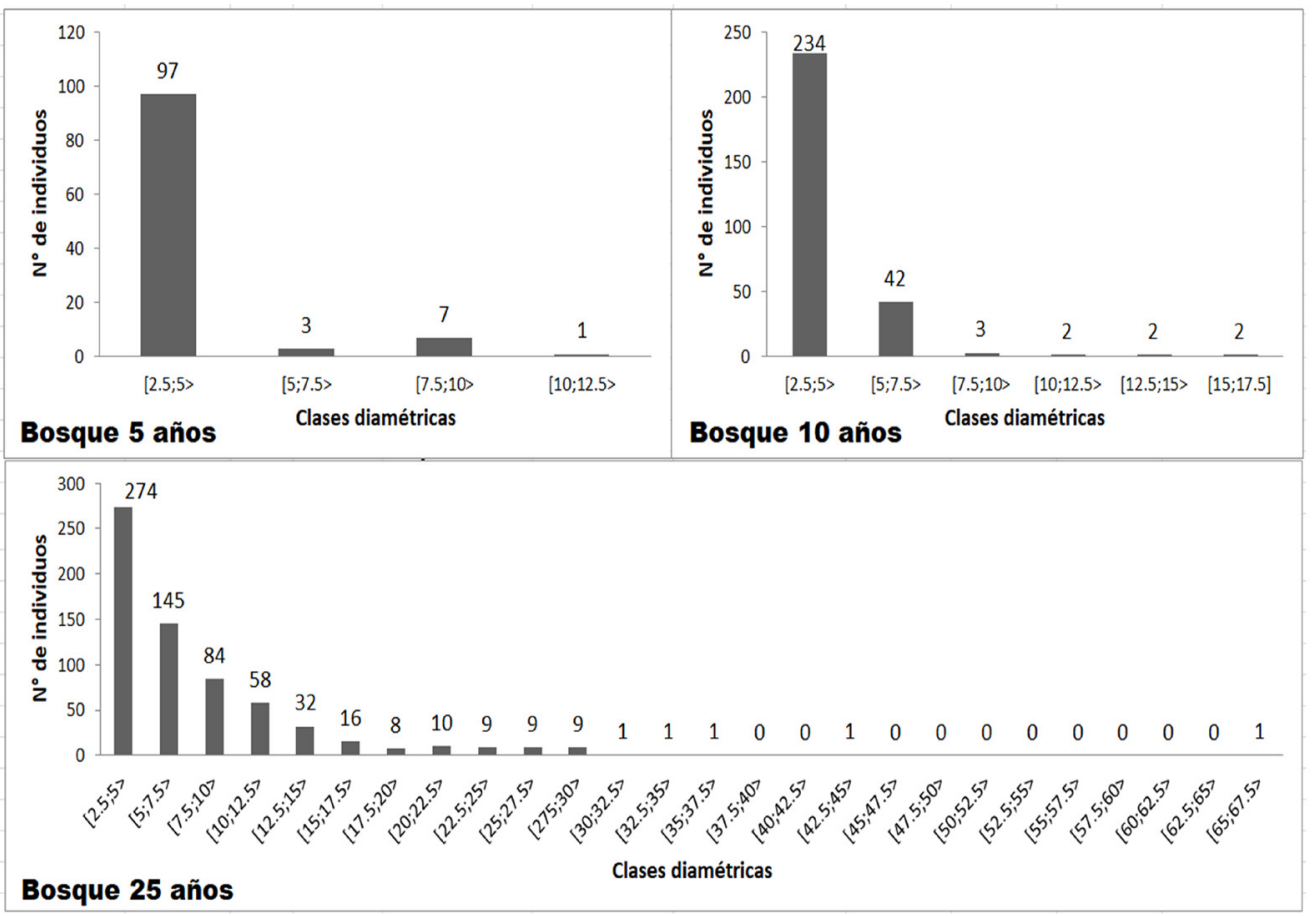

Figura 3. Distribución diamétrica de las diferentes edades de bosque. 


\begin{tabular}{|c|c|c|c|c|c|c|c|c|c|}
\hline \multirow{12}{*}{$\begin{array}{l}\cong \\
\stackrel{\Xi}{0} \\
\stackrel{0}{0}\end{array}$} & Primario & $\begin{array}{c}\text { Phillips } \\
\text { y Miller } \\
\text { (2002) }\end{array}$ & $\stackrel{n}{m}$ & ' & ' & $\stackrel{n}{m}$ & ' & $\stackrel{n}{m}$ & 先 \\
\hline & $>50$ años & $\begin{array}{c}\text { Quintero } \\
\text { (2017) }\end{array}$ & $\stackrel{\circ}{\circ}$ & $\underset{\infty}{+}$ & ठิ & రิ & $\begin{array}{l}\infty \\
\dot{0} \\
I\end{array}$ & $\widehat{\delta}$ & $\hat{8}$ \\
\hline & 40 años & $\begin{array}{c}\text { Quintero } \\
\text { (2017) }\end{array}$ & $\mathscr{\infty}$ & $\underset{i}{\infty}$ & గ్రి & $\hat{\infty}$ & 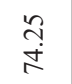 & हెర & $\widetilde{్ ర ి}$ \\
\hline & 30 años & $\begin{array}{c}\text { Quintero } \\
\text { (2017) }\end{array}$ & $\overline{8}$ & 8 & \& & $\underset{\Phi}{\mp}$ & $\tilde{i}$ & $\frac{t}{\sigma}$ & $\frac{\pi}{6}$ \\
\hline & 25 años & $\begin{array}{l}\text { Presente } \\
\text { Estudio }\end{array}$ & $\underset{⿱}{\stackrel{\Delta}{\Delta}}$ & $\overline{\vec{v}}$ & I & જิ & ڤ̊ & సి & స్ \\
\hline & 20 años & $\begin{array}{c}\text { Quintero } \\
\text { (2017) }\end{array}$ & $\vec{\sigma}$ & $\vec{F}$ & $\stackrel{\circ}{F}$ & $\underset{\infty}{\infty}$ & $\begin{array}{l} \pm \\
\pm\end{array}$ & ì & î \\
\hline & \multirow{2}{*}{15 años } & $\begin{array}{l}\text { Quintero } \\
\text { (2017) }\end{array}$ & $\stackrel{\text { }}{\hat{\sim}}$ & ' &, & , & ' & ڤ̊) & ڤิ \\
\hline & & $\begin{array}{l}\text { Cáceres } \\
\text { (2005) }\end{array}$ & $\infty$ & 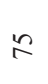 & ' & $n$ & $\stackrel{\leftrightarrow}{\sigma}$ & 2 & 8 \\
\hline & \multirow{2}{*}{10 años } & $\begin{array}{l}\text { Cáceres } \\
\text { (2005) }\end{array}$ & ช్ & ta & ' & $\stackrel{\text { ㄴ }}{=}$ & $\underset{f}{F}$ & 3 & \multirow{2}{*}{2} \\
\hline & & $\begin{array}{l}\text { Presente } \\
\text { Estudio }\end{array}$ & 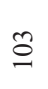 & $n$ & ळ & $\stackrel{\mathscr{\infty}}{\sim}$ & $\overrightarrow{\stackrel{N}{N}}$ & $a$ & \\
\hline & \multirow{2}{*}{5 años } & $\begin{array}{l}\text { Cáceres } \\
\text { (2005) }\end{array}$ & $\vec{\sim}$ & $\approx$ & 1 & f & $\begin{array}{l}\mathscr{\infty} \\
\stackrel{i}{i}\end{array}$ & $\tilde{\lambda}$ & \multirow{2}{*}{ లి } \\
\hline & & $\begin{array}{l}\text { Presente } \\
\text { Estudio }\end{array}$ & i & $\mathscr{\gamma}$ & $m$ & $\stackrel{\infty}{\circ}$ & ?ֶ. & i & \\
\hline & & & 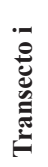 & 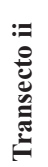 & 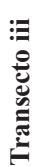 & हूँ & 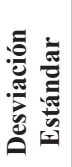 & :气 & 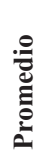 \\
\hline
\end{tabular}


La flora leñosa establecida luego de las quemas en el valle de

mayor diámetro, y para el bosque de 25 años, el $92.4 \%$ se encuentra entre 2.5 y $17,5 \mathrm{~cm}$ de diámetro, siendo Ficus insipida Willd. (65.4 $\mathrm{cm}$ ) la especie de mayor diámetro (Figura 3).

En cuanto a las alturas mínimas y máximas, se aprecia que para el bosque de 5 años estas fueron de $1.5 \mathrm{~m}$ y $6.5 \mathrm{~m}$; para el bosque de 10 años fueron $1.7 \mathrm{~m}$ y $12.5 \mathrm{~m}$; y para el bosque de 25 años fueron $1.5 \mathrm{~m}$ y $16 \mathrm{~m}$ respectivamente.

El área basal registrado para el bosque de 5 años fue $0.13 \mathrm{~m}^{2}$; para el bosque de 10 años fue $0.44 \mathrm{~m}^{2}$; y para el bosque de 25 años fue 5.36 $\mathrm{m}^{2}$.

\section{Discusión}

\section{Riqueza y Composición Florística}

Como se esperaba, el mayor número de especies y riqueza fue encontrado en el bosque de 25 años en comparación a los bosques de 5 y 10 años, estos resultados son consistentes con los reportados por Costa et al. (2017), quienes encontraron mayor riqueza de especies en áreas de 25 años después del fuego que en áreas de 14 años después del fuego.

Para los bosques evaluados con edades de 5 y 10 años, la recuperación en el número de especies sucede de manera más lenta que en bosques originados a partir de otro tipo de alteración como los evaluados por Cáceres (2005) (áreas que posiblemente requirieron una quema inicial para la instalación de cultivos permanentes con limpieza periódica de la vegetación emergente y que posteriormente fueron abandonadas). Del mismo modo se observa un número menor de especies en el bosque de 25 años en comparación a los bosques de 20 y 30 años evaluados por Quintero (2017). Esto indicaría que la recuperación de la riqueza sucede más rápidamente en áreas que fueron abiertas con quema, instalación de agricultura y posterior abandono, en comparación a áreas que fueron sometidas a quemas recurrentes. Posiblemente, porque la materia orgánica y la estructura del suelo no son tan afectadas; y las condiciones de disponibilidad de semillas, vectores de semilla, cantidad de rebrotes y retoños, microclima y condiciones del suelo son más favorables en comparación a las áreas sometidas a quemas recurrentes (Porter y Borgens 1993, Moreira et al. 2010) (Cuadros 2 y 3 ).

En cuanto a las familias y géneros registrados en los bosques con edades de 5 y 10 años, se observa también un menor número de estos en áreas de quemas recurrentes. Asimismo, se observa que en bosques de 15 años (Cáceres 2005) y 20 años (Quintero 2017) se presentan valores cercanos y superiores respectivamente, al ser comparados con el bosque de 25 años del presente estudio. Estas diferencias se deberían posiblemente también a las mismas razones sugeridas anteriormente por Porter y Borgens (1993) y Moreira et al. (2010).

En el bosque evaluado de 25 años, se registró una regeneración significativa del 57\%, del número de familias y géneros presentes en el bosque. Sin embargo en los bosques originados a partir de otro tipo de alteración, dicha recuperación puede tomar solo 15 años (Figura 4 y 5).

\section{Abundancia}

Se observó para las edades iniciales de la sucesión (5-10 años) una diferencia en la composición de familias, géneros y especies, con respecto a aquellos bosques que fueron alteradas por limpieza de la vegetación emergente sin quema (Cáceres 2005). En el primer caso, los taxones más abundantes, en orden decreciente fueron Machaerium inundatum (Mart. ex Benth.) Ducke, Vernonanthura patens (Kunth) H.Rob., Dendrophorbium sp. y Acalypha sp. En el segundo caso, dichos taxones fueron Juglans neotropica Diels, Piper aduncum L., Acalypha macrostachya Jacq. y Guazuma ulmifolia Lam. (Cuadro 4 y 5).

La regeneración, respecto al número de individuos, podría ser mayor en un área sometida a quemas recurrentes que en áreas alteradas por la agricultura en abandono (Lamprecht 1990). Además, Numata et al. (2017) indican que en bosques con edades de 4 y 9 años posteriores a la quema existe una rápida recuperación. Sin embargo, Quintero (2017) indica que el número de individuos es superior en un bosque de 


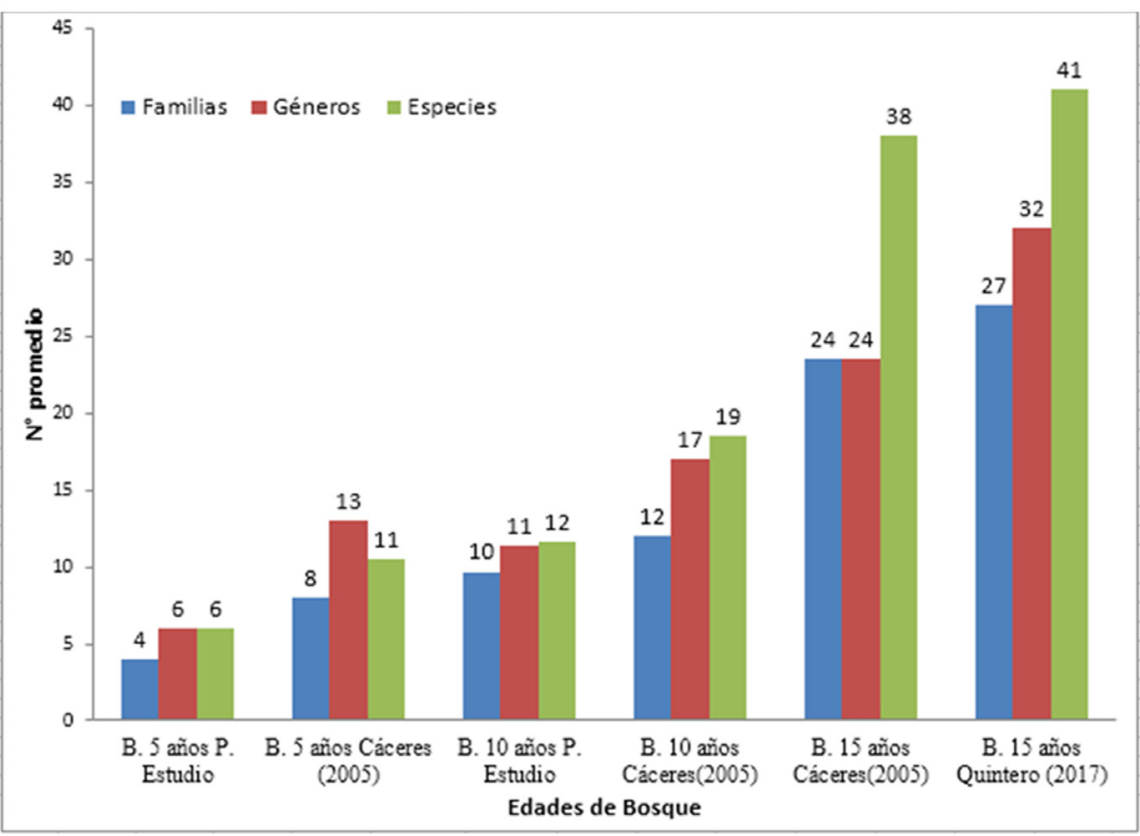

Figura 4. Comparativa en el número de familias, géneros y especies en diferentes edades de bosque.

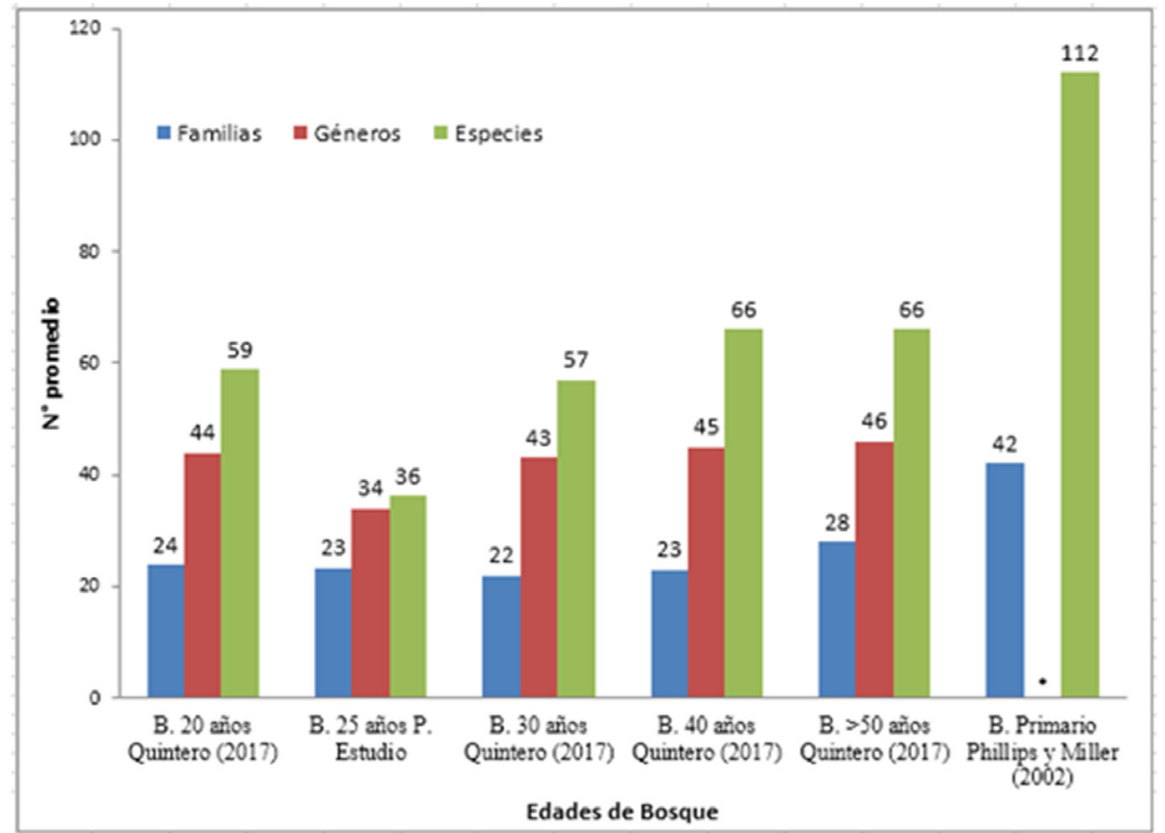

Figura 5. Comparativa en el número de familias, géneros y especies en diferentes edades de bosque. 


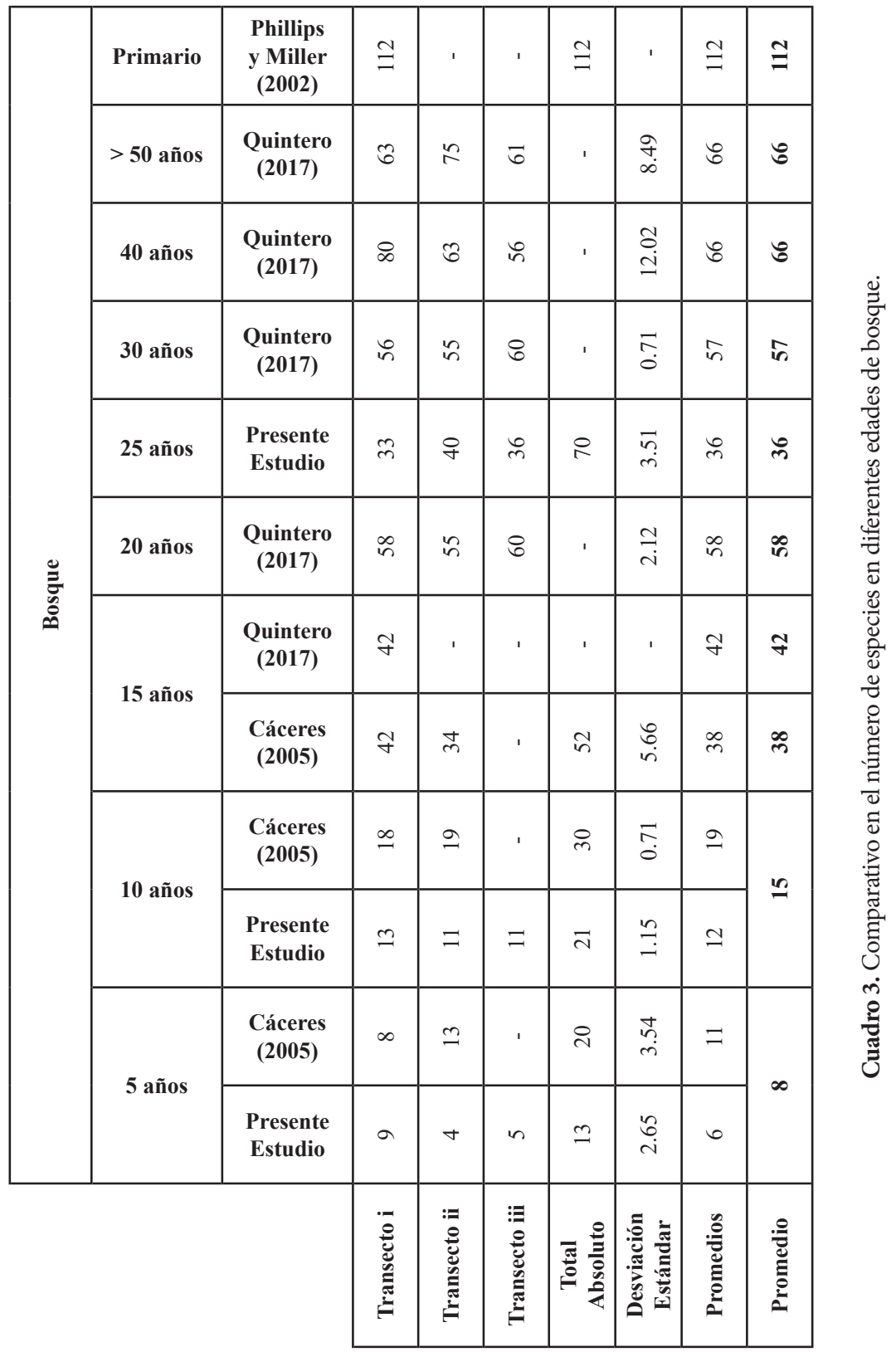




\begin{tabular}{|c|c|c|c|c|}
\hline 롫 & Estudio & Especies & Géneros & Familias \\
\hline \multirow{10}{*}{ 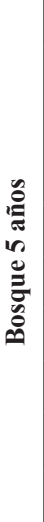 } & \multirow{4}{*}{$\begin{array}{c}\text { Presente } \\
\text { Estudio }\end{array}$} & Vernonanthura patens (65 ind.) & Vernonanthura (65 ind.) & Asteraceae (71 ind.) \\
\hline & & Acalypha sp. (9 ind.) & Acalypha (9 ind.) & Euphorbiaceae (9 ind.) \\
\hline & & Celtis pubescens (8 ind.) & Celtis ( 8 ind.) & Cannabaceae (8 ind.) \\
\hline & & Machaerium inundatum (6 ind.) & Machaerium (6 ind.) & Fabaceae ( 7 ind.) \\
\hline & \multirow{6}{*}{$\begin{array}{c}\text { Cáceres } \\
(2005)\end{array}$} & Guazuma ulmifolia (8 ind.) & Guazuma (8 ind.) & Fabaceae (9 ind.) \\
\hline & & Juglans neotropica (6 ind.) & Persea (8 ind.) & Lauraceae ( 8 ind.) \\
\hline & & Inga setosa (4 ind.) & Juglans (6 ind.) & Malvaceae ( 8 ind.) \\
\hline & & Cecropia polystachya (4 ind.) & Inga (5 ind.) & Juglandaceae (6 ind.) \\
\hline & & Persea boliviana (4 ind.) & & \\
\hline & & Persea sp. (4 ind.) & & \\
\hline \multirow{8}{*}{ 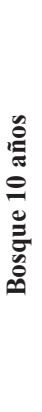 } & \multirow{4}{*}{$\begin{array}{l}\text { Presente } \\
\text { Estudio }\end{array}$} & Machaerium inundatum (103 ind.) & Machaerium (104 ind.) & Fabaceae (109 ind.) \\
\hline & & Dendrophorbium sp. (50 ind.) & Dendrophorbium (50 ind.) & Asteraceae (65 ind.) \\
\hline & & Trema micrantha (31 ind.) & Trema (31 ind.) & Cannabaceae (31 ind) \\
\hline & & Piper aduncum (16 ind.) & Piper (16 ind.) & Euphorbiaceae (16 ind.) \\
\hline & \multirow{4}{*}{$\begin{array}{c}\text { Cáceres } \\
(2005)\end{array}$} & Juglans neotropica (18 ind.) & Piper (24 ind.) & Piperaceae (24 ind.) \\
\hline & & Piper aduncum (18 ind.) & Juglans (18 ind.) & Fabaceae (21 ind.) $\mathrm{xxx}$ \\
\hline & & Acalypha macrostachya (14 ind.) & Acalypha (18 ind.) & Euphrobiaceae (19 ind.) \\
\hline & & Persea boliviana (13 ind.) & Persea (13 ind.) & Juglandaceae (18 ind.) \\
\hline \multirow{10}{*}{ 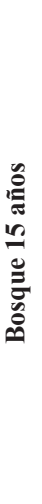 } & \multirow{4}{*}{$\begin{array}{c}\text { Cáceres } \\
(2005)\end{array}$} & Piper aduncum (18 ind.) & Piper (32 ind.) & Piperaceae (32 ind.) \\
\hline & & Acalypha macrostachya (11 ind.) & Acalypha (14 ind.) & Fabaceae (17 ind.) \\
\hline & & Guazuma ulmifolia (10 ind.) & Guazuma (10 ind.) & Euphorbiaceae (15 ind.) \\
\hline & & Piper sp.2 (10 ind.) & Cecropia (9 ind.) & Malvaceae (10 ind.) \\
\hline & \multirow{6}{*}{$\begin{array}{c}\text { Quintero } \\
\text { (2017) }\end{array}$} & Piper hispidum (39 ind.) & Piper (50 ind.) & Piperaceae (50 ind.) \\
\hline & & Chamaedorea linearis (16 ind.) & Chamaedorea (16 ind.) & Euphorbiaceae (26 ind.) \\
\hline & & Acalypha macrostachya (13 ind.) & Acalypha (13 ind.) & Arecaceae (16 ind.) \\
\hline & & Sapium glandulosum (13 ind.) & Sapium (13 ind.) & Rubiaceae (16 ind.) \\
\hline & & Lacistema aggregatum (11 ind.) & Lacistema (11 ind.) & Fabaceae (12 ind.) \\
\hline & & Toxicodendron striatum (11 ind.) & Toxicodendron (11 ind.) & Malvaceae (12 ind.) \\
\hline
\end{tabular}

Cuadro 4. Comparativo de la abundancia en la composición por familias, géneros y especies en las diferentes edades de bosque (i). 


\begin{tabular}{|c|c|c|c|c|}
\hline ్ㅠㄹ & Estudio & Especies & Géneros & Familias \\
\hline 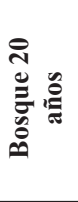 & $\begin{array}{c}\text { Quintero } \\
\text { (2017) }\end{array}$ & $\begin{array}{l}\text { Trophis caucana (157 ind.) } \\
\text { Piper reticulatum (133 ind.) } \\
\text { Piper aduncum (106 ind.) } \\
\text { Cecropia obtusifolia (74 ind.) }\end{array}$ & $\begin{array}{l}\text { Piper (299 ind.) } \\
\text { Trophis (157 ind.) } \\
\text { Cecropia }(90 \text { ind.) } \\
\text { Miconia ( } 78 \text { ind.) }\end{array}$ & $\begin{array}{l}\text { Piperaceae (303 ind.) } \\
\text { Urticaceae ( } 230 \text { ind.) } \\
\text { Moraceae (167 ind.) } \\
\text { Fabaceae (106 ind.) }\end{array}$ \\
\hline 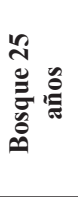 & $\begin{array}{l}\text { Presente } \\
\text { Estudio }\end{array}$ & $\begin{array}{l}\text { Heteropterys laurifolia (75 ind.) } \\
\text { Piper aduncum (65 ind.) } \\
\text { Allophylus floribundus (55 ind.) } \\
\text { Sapium glandulosum (53 in.) }\end{array}$ & $\begin{array}{l}\text { Piper (96 ind.) } \\
\text { Heteropterys ( } 75 \text { ind.) } \\
\text { Allophylus (55 ind.) } \\
\text { Sapium (53 ind.) }\end{array}$ & $\begin{array}{l}\text { Piperaceae (96 ind.) } \\
\text { Euphorbiaceae ( } 80 \text { ind.) } \\
\text { Malpighiaceae ( } 79 \text { ind.) } \\
\text { Sapindaceae (64 ind.) }\end{array}$ \\
\hline 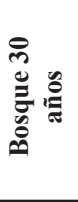 & $\begin{array}{c}\text { Quintero } \\
\text { (2017) }\end{array}$ & $\begin{array}{l}\text { Trophis caucana (308 ind.) } \\
\text { Piper reticulatum (237 ind.) } \\
\text { Piptadenia klugii (186 ind.) } \\
\text { Urera laciniata (59 ind.) }\end{array}$ & $\begin{array}{l}\text { Trophis (308 ind.) } \\
\text { Piper (281 ind.) } \\
\text { Piptadenia (186 ind.) } \\
\text { Urera (143 ind.) }\end{array}$ & $\begin{array}{l}\text { Moraceae (360 ind.) } \\
\text { Urticaceae (282 ind.) } \\
\text { Piperaceae ( } 281 \text { ind.) } \\
\text { Fabaceae ( } 275 \text { ind.) }\end{array}$ \\
\hline 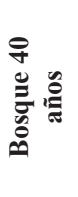 & $\begin{array}{c}\text { Quintero } \\
\text { (2017) }\end{array}$ & $\begin{array}{l}\text { Trophis caucana (137 ind.) } \\
\text { Guarea guidonia (107 ind.) } \\
\text { Cespedesia spathulata (101 ind.) } \\
\text { Boehmeria caudata (97 ind.) }\end{array}$ & $\begin{array}{l}\text { Guarea (139 ind.) } \\
\text { Trophis (137 ind.) } \\
\text { Piper (120 ind.) } \\
\text { Ocotea (108 ind.) }\end{array}$ & $\begin{array}{l}\text { Moraceae (236 ind.) } \\
\text { Urticaceae ( } 222 \text { ind.) } \\
\text { Lauraceae (161 ind) } \\
\text { Arecaceae (158 ind.) }\end{array}$ \\
\hline 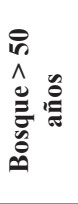 & $\begin{array}{c}\text { Quintero } \\
\text { (2017) }\end{array}$ & $\begin{array}{l}\text { Trophis caucana (471 ind.) } \\
\text { Neea macrophylla (135 ind.) } \\
\text { Guarea guidonia (102 ind.) } \\
\text { Ocotea cernua (94 ind.) }\end{array}$ & $\begin{array}{l}\text { Trophis (471 ind.) } \\
\text { Ocotea (210 ind.) } \\
\text { Guarea (170 ind.) } \\
\text { Neea (135 ind.) }\end{array}$ & $\begin{array}{l}\text { Moraceae (633 ind.) } \\
\text { Lauraceae ( } 316 \text { ind.) } \\
\text { Meliaceae (170 ind.) } \\
\text { Nyctaginaceae (135 ind.) }\end{array}$ \\
\hline 芑 & $\begin{array}{c}\text { Phillips } \\
\text { y Miller } \\
\text { (2002) }\end{array}$ & $\begin{array}{l}\text { Phytelephas sp.1 (55 ind.) } \\
\text { Acalypha diversifolia (27 ind.) } \\
\text { Drypetes sp.1 (23 ind) } \\
\text { Prunus sp.1 (10 ind) }\end{array}$ & & $\begin{array}{l}\text { Moraceae (61 ind.) } \\
\text { Fabaceae (52 ind.) } \\
\text { Sapindaceae (41 ind.) } \\
\text { Lauraceae ( } 34 \text { ind.) }\end{array}$ \\
\hline
\end{tabular}

Cuadro 4. Comparativo de la abundancia en la composición por familias, géneros y especies en las diferentes edades de bosque (ii).

20 años en comparación con el bosque de 25 años del presente estudio. Indicando además que la regeneración y sucesión de los bosques es mayor en áreas que en el pasado fueron dedicadas a la agricultura (expuestas a quemas y luego abandonadas) que en áreas expuestas a quemas frecuentes. Esto posiblemente debido a que las condiciones de cantidades suficien- tes de semillas viables, las condiciones (micro) climáticas y edáficas adecuadas para la germinación y desarrollo fueron más favorables en el bosque de 20 años (Lamprecht 1990). Además, se observa en los bosques secundarios evaluados por Quintero (2017) una relación directamente proporcional de la edad con el número de individuos. 


\section{Estructura}

Se observa que los valores correspondientes a los diámetros, alturas y áreas basales se incrementan gradualmente desde los estadíos iniciales de la sucesión (5, 10, 25 años). Además, para todas las edades de bosque se observa una distribución diamétrica con un mayor número de individuos en las primeras clases, resultados similares han sido reportados por Costa et al. (2017).

Los valores de diámetro promedio para las edades de bosque secundario de 5, 10 y 25 años son inferiores a los valores encontrados por Cáceres (2005) en los bosques secundarios de 5, 10 y 15 años. Estas diferencias posiblemente se deban a que la quema influye directamente en la disponibilidad de nutrientes, pues si bien es cierto que la disponibilidad de éstos al inicio en el área sometida a quema es mayor por las cenizas presentes, estas disminuyen al pasar el tiempo por efectos de la erosión hídrica y eólica (Anderson y Spencer 1992), además se debe tener en cuenta el efecto de la topografía en estos bosques sometidos a quemas, principalmente en los de 10 y 25 años de edad, donde la pendiente es pronunciada (mayor a $50 \%$ ). Y comparativamente las áreas sometidas a limpieza y sin quema, se presenta materia orgánica disponible en descomposición y por un tiempo más prolongado (Moreira et al. 2010).

\section{Conclusiones}

Entre los 10-25 años de edad del bosque se produce una recomposición importante de la riqueza (57\% de familias del bosque primario), lo que posiblemente implique la recuperación del bosque.

En áreas sometidas a quemas recurrentes, el establecimiento de la regeneración es relativamente más denso que en áreas originadas de cultivos permanentes abandonados (que posiblemente requirieron una quema inicial para su instalación), pero inferior en áreas que en el pasado fueron dedicadas a la agricultura (cultivos de café, expuestos a las quemas y luego abandonados).
Para los bosques originados desde quemas, $y$ en estadios tempranos (5-10 años), se produce un mayor número de individuos y menor número de especies, géneros y familias que en bosques secundarios (de similares edades) originados de cultivos permanentes abandonados (que posiblemente requirieron una quema inicial para su instalación).

Asimismo, a partir de los 15 años de edad, los bosques secundarios (de diferentes condiciones iniciales de formación) alcanzan relativa similitud en el número de familias, géneros $\mathrm{y}$ especies.

\section{Agradecimientos}

Al Herbario Forestal MOL y al personal del IRD - La Génova por las facilidades logísticas durante las labores de campo. A Robin Fernandez, Ítalo Revilla y Rosa Villanueva por su colaboración para completar la identificación de varias de las especies colectadas. A la Asociación Peruana para la Promoción del Desarrollo Sostenible (APRODES) por el apoyo financiero parcial para completar el presente trabajo.

\section{Bibliografía}

Anderson, J; Spencer, T. 1991. Carbon, nutrient and water balances of tropical rain forest ecosystem subject to disturbances: management implications and research proposals. París, Francia. 95 p.

Armenteras, D; Rodríguez, N. 2014. Dinámicas y causas de deforestación en bosques de Latino América: Una revisión desde 1990. Colombia Forestal 17(2):233-246.

Bridson, D; Forman, L. 1998. The herbarium handbook. Royal Botanical Garden, Kew, England. $334 \mathrm{p}$.

Cáceres, P. 2004. Caracterización dendrológica de las especies de los géneros Ficus y Cecropia (Moraceae) en el valle de Chanchamayo (Junín-Perú). Tesis Ing. Forestal. Lima, Perú. Universidad Nacional Agraria La Molina. 337p.

Cáceres, B. 2005. Diversidad de la composición florística de la microcuenca de Santa Rosa, 
La flora leñosa establecida luego de las quemas en el valle de

Chanchamayo, Junín. Tesis Mg. Sc. Ciencias Ambientales. Lima, Perú. Universidad Nacional Agraria la Molina. 241 p.

Costa, M; De Meneses, L; Nascimento, M. 2017. Post-Fire regeneration in seasonally dry tropical forest fragments in southeastern Brazil. Anais da academia Brasileira de Ciencias 89(4):2687-2695.

De La Torre, C. 2002. Caracterización dendrológica y claves de identificación de Lauráceas en el Valle de Chanchamayo (Junín - Perú). Tesis Ing. Forestal. Lima, Perú. Universidad Nacional Agraria La Molina. 224p.

Gentry, A. 1982. Patterns of neotropical plant species diversity. Evolutionary biology 15:1-84.

Numata, I; Silva, S; Cochrane, M; d'Oliveira, M. 2017. Fire and edge effects in a fragmented tropical forest landscape in the southwestern Amazon. Forest Ecology and Management 401(2017):135-146.

Gómez-Pompa, A; Burley, F. 1991. The Management of natural tropical forests. In Gómez-Pompa, A; Whitmore, TC; Hadley, M (eds). Rain forest regeneration and management. Vol. 6 (Man and the Biosphere Series).

Lamprecht, H. 1990. Silvicultura en los trópicos: los ecosistemas forestales en los bosques tropicales y sus especies arbóreas-posibilidades y métodos para un aprovechamiento sostenido. Trad. A Carrillo. Eschborn, Deutschland. $335 \mathrm{p}$.

Macbride, F. 1936-1971. Flora of Peru. Publ. Field Mus. Nat. Hist., Botanical Series, Vol 13.

Manta, M. 2005. Evaluación de los incendios forestales en la provincia de Satipo, Departamento de Junín, Perú. Programa de las Naciones Unidas para el Desarrollo (PNUD) / Universidad Nacional Agraria La Molina. Lima, Perú. 58 p.

Manta, M. 2007. Silvicultura de bosques naturales. Curso de nivel graduado. Escuela de post grado. Programa de Bosques y Gestión de Recursos Forestales. Universidad Nacional Agraria La Molina. Lima, Perú. 1200 diapositivas.
Martinez, G; Hernández, M; Ordoñez, J; Camacho, J. 2013. Régimen y distribución de los incendios forestales en el estado de México (2000 a 2011). Revista Mexicana de Ciencias Forestales 6(29):92-107.

Meneses, E. 1989. Identificación y caracterización dendrológica de 15 especies forestales Leguminosas de la zona de Chanchamayo. Tesis Ing. Forestal. Lima, Perú. Universidad Nacional Agraria La Molina. 101 p.

Moreira, F; Catry, F; Silva, J; Rego, F. 2010. Ecologia do fogo e gestão de áreas ardidas. Lisboa, Portugal. $322 \mathrm{p}$.

Phillips, O; Miller, J. 2002. Global patterns of plant diversity: Alwyn H. Gentry's forest transect data set. Monographs in systematic Botany from the Missouri Botanical Garden. St. Louis, Missouri. United States. 319 p.

Pino, D. 2002. Caracterización dendrológica de las Rubiáceas de los bosques de Chanchamayo, Junín. Tesis Ing. Forestal. Lima, Perú. Universidad Nacional Agraria La Molina. 212 p.

Poorter, L; Borgens, F. 1993. Ecology of tropical forests. Departament of Forestry. Wageningen Agricultural University. 223 p.

Quintero, F. 2017. Características de la sucesión Vegetal en el valle de Chanchamayo y sus implicancias para su conservación y manejo forestal. Tesis Msc. Lima, Perú. Universidad Nacional Agraria La Molina. 356 p.

Reynel, C; Pennington, T.D; Pennington. R.T. 2016. Árboles del Perú. Lima, Imprenta Bellido, 1047 p.

Reynel, C; Pennington, R; Pennington, T; Marcelo, J; Daza, A. 2007. Árboles útiles del Ande peruano y sus usos: un manual con apuntes de identificación, ecología y propagación de las especies de la Sierra y los Bosques Montanos en el Perú. Herbario de la Facultad de Ciencias Forestales de la Universidad $\mathrm{Na}-$ cional Agraria La Molina, Royal Botanic Gardens Kew, Royal Botanic Gardens Edinburgh, APRODES. 463 p.

Silva, M. 2005. Estudio y caracterización dendrológica de las familias botánicas del orden 
Malvales en el Fundo la Génova (Chanchamayo). Tesis Ing. Forestal. Lima, Perú. Universidad Nacional Agraria La Molina. 238 p.

Spichiger, R; Méroz, J; Loizeau. PA; Stutz de Ortega, L. 1990. Contribución a la flora de la Amazonía Peruana. Los árboles del arborétum Jenaro Herrera. 358 p. 
Anexos

Anexo 1. Abundancia en la composición florística en el bosque de 5 años.

\begin{tabular}{|c|c|l|c|}
\hline $\mathbf{N}^{\circ}$ & Familia & \multicolumn{1}{|c|}{ Especie } & $\mathbf{N}^{\circ}$ de individuos \\
\hline 1 & Fabaceae & Machaerium inundatum (Mart. ex Benth.) Ducke & 103 \\
\hline 2 & Asteraceae & Dendrophorbium sp. & 50 \\
\hline 3 & Cannabaceae & Trema micrantha (L.) Blume & 31 \\
\hline 4 & Piperaceae & Piper aduncum L. & 16 \\
\hline 5 & Euphorbiaceae & Sapium glandulosum (L.) Morong & 15 \\
\hline 6 & Asteraceae & Cyrtocymura scorpioides (Lam.) H. Rob. & 13 \\
\hline 7 & Urticaceae & Cecropia polystachya Trécul & 9 \\
\hline 8 & Melastomataceae & Miconia dipsacea Naudin & 8 \\
\hline 9 & Rubiaceae & Chomelia paniculata (Bartl. ex DC.) Steyerm. & 8 \\
\hline 10 & Primulaceae & Myrsine pellucida (Ruiz \& Pav.) Spreng. & 7 \\
\hline 11 & Anacardiaceae & Mauria heterophylla Kunth & 5 \\
\hline 12 & Fabaceae & Inga sp. & 5 \\
\hline 13 & Myrtaceae & Psidium guajava L. & 3 \\
\hline 14 & Solanaceae & Solanum sp. & 3 \\
\hline 15 & Malvaceae & Ochroma pyramidale (Cav. ex Lam.) Urb. & 2 \\
\hline 16 & Salicaceae & Banara guianensis Aubl. & 2 \\
\hline 17 & Asteraceae & Pappobolus sp. & 1 \\
\hline 18 & Asteraceae & Vernonanthura patens (Kunth) H. Rob. & 1 \\
\hline 19 & Euphorbiaceae & Acalypha sp. & 1 \\
\hline 20 & Fabaceae & Machaerium acutifolium Vogel & 1 \\
\hline 21 & Rubiaceae & Indeterminado sp. 2 & 1 \\
\hline & & & \\
\hline
\end{tabular}


Anexo 2. Abundancia en la composición florística en el bosque de 10 años.

\begin{tabular}{|c|c|l|c|}
\hline $\mathbf{N}^{\circ}$ & Familia & \multicolumn{1}{|c|}{ Especie } & No de individuos \\
\hline 1 & Asteraceae & Vernonanthura patens (Kunth) H. Rob. & 65 \\
\hline 2 & Euphorbiaceae & Acalypha sp. & 9 \\
\hline 3 & Cannabaceae & Celtis pubescens Spreng. & 8 \\
\hline 4 & Fabaceae & Machaerium inundatum (Mart. ex Benth.) Ducke & 6 \\
\hline 5 & Piperaceae & Piper heterophyllum Ruiz \& Pav. & 5 \\
\hline 6 & Urticaceae & Cecropia polystachya Trécul & 5 \\
\hline 7 & Asteraceae & Indeterminado sp.1 & 3 \\
\hline 8 & Asteraceae & Lepidaploa aff. canescens (Kunth) H. Rob. & 2 \\
\hline 9 & Asteraceae & Monactis sp. & 1 \\
\hline 10 & Fabaceae & Erythrina ulei Harms & 1 \\
\hline 11 & Solanaceae & Cestrum sp. & 1 \\
\hline 12 & Solanaceae & Solanum riparium Pers. & 1 \\
\hline 13 & Urticaceae & Urera caracasana (Jacq.) Gaudich. ex Griseb. & 1 \\
\hline
\end{tabular}


Anexo 3. Abundancia en la composición florística en el bosque de 25 años (i).

\begin{tabular}{|c|c|c|c|}
\hline $\mathbf{N}^{\circ}$ & Familia & Especie & $\mathbf{N}^{\circ}$ de individuos \\
\hline 1 & Malpighiaceae & Heteropterys laurifolia (L.) A. Juss. & 75 \\
\hline 2 & Piperaceae & Piper aduncum L. & 65 \\
\hline 3 & Sapindaceae & Allophylus floribundus (Poepp.) Radlk. & 55 \\
\hline 4 & Euphorbiaceae & Sapium glandulosum (L.) Morong & 53 \\
\hline 5 & Anacardiaceae & Mauria heterophylla Kunth & 41 \\
\hline 6 & Fabaceae & Machaerium inundatum (Mart. ex Benth.) Ducke & 37 \\
\hline 7 & Lauraceae & Persea caerulea (Ruiz \& Pav.) Mez & 30 \\
\hline 8 & Euphorbiaceae & Acalypha sp. & 24 \\
\hline 9 & Moraceae & Trophis caucana (Pittier) C.C. Berg & 24 \\
\hline 10 & Piperaceae & Piper heterophyllum Ruiz \& Pav. & 22 \\
\hline 11 & Urticaceae & Cecropia polystachya Trécul & 17 \\
\hline 12 & Clusiaceae & Clusia sp. & 16 \\
\hline 13 & Melastomataceae & Miconia dipsacea Naudin & 15 \\
\hline 14 & Primulaceae & Myrsine guianensis (Aubl.) Kuntze & 15 \\
\hline 15 & Asteraceae & Dendrophorbium sp. & 10 \\
\hline 16 & Asteraceae & Viguiera sp. & 9 \\
\hline 17 & Lacistemataceae & Lacistema aggregatum (P.J. Bergius) Rusby & 8 \\
\hline 18 & Meliaceae & Guarea guidonia (L.) Sleumer & 8 \\
\hline 19 & Sapindaceae & Cupania cinérea Poepp. & 7 \\
\hline 20 & Lauraceae & Ocotea acyphylla (Nees \& Mart.) Mez & 6 \\
\hline 21 & Rubiaceae & Palicourea macrobotrys (Ruiz \& Pav.) DC. & 6 \\
\hline 22 & Urticaceae & Myriocarpa stipitata Benth. & 6 \\
\hline 23 & Asteraceae & Cyrtocymura scorpioides (Lam.) H. Rob. & 5 \\
\hline 24 & Combretaceae & Terminalia amazonia (J.F. Gmel.) Exell & 5 \\
\hline 25 & Malpighiaceae & Bunchosia argentea (Jacq.) DC. & 5 \\
\hline 26 & Piperaceae & Piper hispidum Sw. & 5 \\
\hline 27 & Urticaceae & Urera caracasana (Jacq.) Gaudich. ex Griseb. & 5 \\
\hline 28 & Piperaceae & Piper formosum (Miq.) C. DC. & 4 \\
\hline 29 & Proteaceae & Roupala montana Aubl. & 4 \\
\hline 30 & Rhamnaceae & Rhamnus sp. & 4 \\
\hline 31 & Rubiaceae & Randia armata (Sw.) DC. & 4 \\
\hline 32 & Rubiaceae & Simira sp. & 4 \\
\hline 33 & Cannabaceae & Trema micrantha $(\mathrm{L}$.$) Blume$ & 3 \\
\hline 34 & Malvaceae & Ochroma pyramidale (Cav. ex Lam.) Urb. & 3 \\
\hline 35 & Moraceae & Batocarpus costaricensis Standl. \& L.O. Williams & 3 \\
\hline
\end{tabular}


Anexo 4. Abundancia en la composición florística en el bosque de 25 años (ii).

\begin{tabular}{|c|c|c|c|}
\hline $\mathbf{N}^{\circ}$ & Familia & Especie & $\mathbf{N}^{\circ}$ de individuos \\
\hline 36 & Moraceae & Ficus cuatrecasasiana Dugand & 3 \\
\hline 37 & Myrtaceae & Eugenia uniflora $\mathrm{L}$. & 3 \\
\hline 38 & Salicaceae & Casearia decandra Jacq. & 3 \\
\hline 39 & Calophyllaceae & Calophyllum brasiliense Cambess. & 3 \\
\hline 40 & Annonaceae & Cymbopetalum sp & 2 \\
\hline 41 & Asteraceae & aff. Clibadium sp. & 2 \\
\hline 42 & Asteraceae & Oyedaea rusbyi S.F. Blake & 2 \\
\hline 43 & Euphorbiaceae & Croton $\mathrm{sp}$. & 2 \\
\hline 44 & Fabaceae & Erythrina ulei Harms & 2 \\
\hline 45 & Moraceae & Ficus insipida Willd. & 2 \\
\hline 46 & Myrtaceae & Campomanesia lineatifolia Ruiz \& Pav. & 2 \\
\hline 47 & Myrtaceae & Psidium guajava $\mathrm{L}$. & 2 \\
\hline 48 & Nyctaginaceae & Neea parviflora Poepp. \& Endl. & 2 \\
\hline 49 & Polygonaceae & Coccoloba sp. & 2 \\
\hline 50 & Rosaceae & Prunus sp. & 2 \\
\hline 51 & Sapindaceae & Dilodendron bipinnatum Radlk. & 2 \\
\hline 52 & Anacardiaceae & Tapirira guianensis Aubl. & 1 \\
\hline 53 & Aquifoliaceae & Ilex inundata Poepp. ex Reissek & 1 \\
\hline 54 & Asteraceae & Vernonanthura patens (Kunth) H. Rob. & 1 \\
\hline 55 & Boraginaceae & Cordia alliodora (Ruiz \& Pav.) Oken & 1 \\
\hline 56 & Clusiaceae & Calophyllum brasiliense Cambess. & 1 \\
\hline 57 & Combretaceae & Terminalia oblonga (Ruiz \& Pav.) Steud. & 1 \\
\hline 58 & Erythroxylaceae & Erythroxylon $\mathrm{sp}$. & 1 \\
\hline 59 & Juglandaceae & Juglans neotropica Diels & 1 \\
\hline 60 & Lamiaceae & Hyptis sp. & 1 \\
\hline 61 & Lauraceae & Ocotea obovata (Ruiz \& Pav.) Mez & 1 \\
\hline 62 & Malvaceae & Ceiba insignis (Kunth) P.E. Gibbs \& Semir & 1 \\
\hline 63 & Malvaceae & Guazuma ulmifolia Lam. & 1 \\
\hline 64 & Moraceae & Brosimum guianense (Aubl.) Huber & 1 \\
\hline 65 & Moraceae & Clarisia biflora Ruiz \& Pav. & 1 \\
\hline 66 & Moraceae & Ficus pertusa L. f. & 1 \\
\hline 67 & Myrtaceae & Eugenia muricata DC. & 1 \\
\hline 68 & Rubiaceae & Indeterminado sp.1 & 1 \\
\hline 69 & Salicaceae & Banara guianensis Aubl. & 1 \\
\hline 70 & Urticaceae & Pourouma cecropiifolia Mart. & 1 \\
\hline 71 & Adoxaceae & Viburnum sp. & 1 \\
\hline
\end{tabular}

